\title{
Desafíos en la ampliación del tiempo escolar en dos contextos: Distrito Federal (Brasil) y Cataluña (España)
}

\section{The challenge of school time extension in two contexts: Federal District (Brazil) and Catalonia (Spain)}

\author{
Rita de Cássia Oliveira Cruz ${ }^{(i)}$ \\ Remi Castioni (ii)
}

(i) Universidade de Brasília - UnB, Brasília, DF, Brasil. http:/ / orcid.org/0000-0002-1203-4617, cruz11rita@gmail.com.

(ii) Universidade de Brasília - UnB, Brasília, DF, Brasil. https://orcid.org/0000-0002-5459-3492, remi@unb.br, rcastioni@globo.com.

Resumen: El presente artículo se propone analizar la ampliación del tiempo escolar en la educación básica; y evidencia que el tema viene ocupando un gran espacio en la literatura internacional. En Brasil, el mismo pasa a ganar relevancia con la nueva reorganización de la enseñanza secundaria. Por tratarse de un estudio comparado, el artículo se centra en la experiencia de organización del tiempo escolar del Distrito Federal (Brasil) en comparación con Cataluña (España); y debido a diferentes términos y conceptos el estudio se centra en el análisis del tiempo diario de los estudiantes. Así presenta como resultado las diferencias en la organización de la jornada escolar diaria, en la autonomía y en la infraestructura de los centros educativos de los dos contextos. Una conclusión, entre otras, es que la referida ampliación exige la valoración de la opinión de diferentes interlocutores y del contexto donde se desarrollará la propuesta.

Palabras clave: día escolar extendido; jornada escolar; estudio comparado 


\section{pro.posições}

Abstract: The present article contemplates an analysis on the extension of the school time in the basic education; and evidences that the subject has occupied great space in the international literature. In Brazil, he comes to gain relevance with the new reorganization of secondary education. Because this is a comparative study, the article focuses on the experience of organizing school time in the Federal District (Brazil) compared to Catalonia (Spain); and based on the diversity of terms and concepts, the study focuses the analysis of the student's daily time. With that, it presents as results the differences in the organization of the daily school day, in the autonomy and in the infrastructure of the educational centers of the two contexts. And he concludes, among others, that this expansion requires the assessment of the opinions of different interlocutors and the context in which the proposal will be developed.

Keywords: extended school day; time of school; comparative education

\section{Consideraciones iniciales}

La discusión sobre el tema tiempo escolar, en Brasil, parece haber ocupado el centro de la discusión. Ha ganado relevancia en ocasión de los acalorados debates en torno a la reforma de la educación secundaria que puso en marcha, entre otros aspectos, la ampliación del tiempo diario de permanencia de los estudiantes en los institutos. Por ello, abordar esa temática ayuda a ampliar la comprensión en torno al asunto, a partir de una mirada a diferentes experiencias internacionales. En este sentido, el estudio permite comprender los cambios en el papel de la escuela pública brasileña, puesto que ha incorporado otras actividades a lo largo de los años. Pero al mismo tiempo centra la discusión en la organización del tiempo escolar de los estudiantes, a partir de un análisis sobre la experiencia del Distrito Federal (Brasil) en comparación con Cataluña (España), considerando la revisión de la literatura, sobre todo informes de investigación y observación de clases.

Debido a la diversidad de conceptos, el artículo se centra en la idea de tiempo que los estudiantes dedican a diario a su participación en las aulas y en actividades extraescolares. En este sentido, el estudio tiene como categorías de análisis la carga horaria semanal, la organización del tiempo escolar, la doble jornada y la toma de decisión por esa jornada, en los dos contextos; y hace referencia a la diversidad de experiencias respecto al tiempo de la jornada regular, en diferentes países. 


\section{pro.posıções}

\section{Metodología}

En este estudio, de enfoque descriptivo, se empleó el método comparado. Y sobre ello hay que mencionar la existencia de diversas contribuciones que resaltan la cuestión del método (Mason, 2015; Fairbrother, 2015, Schneider \& Schimitt, 1998; Vega Gil, 2011 y otros). En este sentido se entiende método comparado al proceso sistemático de análisis de un fenómeno, problema, sistemas, de distintos países, localidades, siguiendo etapas determinadas (descripción, interpretación, yuxtaposición y comparación), aunque no sea obligatorio cumplir con todas ellas. En este estudio, por ejemplo, se priorizó la descripción, la interpretación y la comparación, y por una cuestión didáctica el texto agrupa la descripción y la interpretación.

Por otra parte, es necesario mencionar que la comparación que se hizo supera el concepto cotidiano de estudios comparados, en el que se prevé la existencia de elementos en común en base a distintos aspectos (cultural, histórico, social, demográfico, etc.). Hay una predominancia de estudios que ponen énfasis en la perspectiva tradicional de estudios comparados, es decir, la ausencia de elementos semejantes, entre los objetos de análisis, se inserta como un aspecto limitador para la comparación. En este sentido, la identificación de problemáticas inherentes y comunes a los dos contextos (Distrito Federal y Cataluña) permitió realizar tal comparación pues se han encontrado elementos que aproximan las dos experiencias y otros que evidencian sus singularidades.

Un elemento que las distingue y que justifica este estudio, en parte, es la prueba de que Brasil es uno de los países, de América Latina, con menor tiempo diario de permanencia de los estudiantes en la escuela, pero también la singular organización de las escuelas/institutos públicos del Distrito Federal (y que se diferencia de los demás estados del país), sumado a la reciente reforma en la educación secundaria propuesta por el gobierno central brasileño, a diferencia de Cataluña, que oferta la secundaria en un único turno. Por ello, este estudio buscó conocer en qué medida la configuración actual de la organización del tiempo escolar, en la educación primaria, del Distrito Federal (Brasil), atiende a las necesidades educativas de los estudiantes.

Se intentó contestar a esta indagación por medio del análisis de la categoría tiempo escolar diario de los estudiantes. El objetivo de describir la organización de la jornada escolar regular, así como la forma de organización de la doble jornada, de escuelas públicas de los dos contextos, a partir del análisis de unidades de comparación (carga horaria semanal, la 


\section{pro.posições}

organización del tiempo escolar y la decisión de la doble jornada). En ese sentido, se aplicó la técnica de análisis de contenido temático para el examen de las referidas categorías (Franco, 2008). De esta manera fue posible describir la experiencia del Distrito Federal y de Cataluña y comparar las peculiaridades en la organización de ese tiempo, en los dos contextos.

Todo el proceso fue fundamentado a partir de las observaciones no participantes, realizadas en dos escuelas públicas de primaria (de los dos contextos), de revisión de la literatura y análisis de documentos base, como informes de investigación. Y sobre estos informes es necesario mencionar que fueron elegidos por representar resultados de investigaciones desarrolladas en la misma década y por ser referencia de estudios sobre el tema, en los dos contextos.

En el caso de Brasil, se eligió el documento presentado por el Ministério de Educação (MEC), de Brasil, en 2009 - intitulado de Educação integral/educação integrada e(m) tempo integral: concepções e práticas na educação brasileira. El documento evidencia resultados de una investigación desarrollada por expertos de cuatro universidades públicas a petición del propio ministerio, con el objetivo, entre otros, de mapear experiencias de ampliación de la jornada escolar en educación primaria y fundamentar propuestas de políticas públicas dirigidas a la educación integral a nivel nacional. Además, para ese contexto, se han tomado como base otras publicaciones (Leclerc \& Moll, 2012; Menegatti \& Valentini, 2012; Rosa, 2011).

Respecto a España (en particular, a la experiencia de Cataluña) se ha tomado como base el estudio de Herrero (2012), intitulado La perspectiva social de la jornada escolar en la Unión Europea: estudio comparado en Italia, Francia, Portugal y España; investigación realizada a partir de un análisis de experiencias de estos países, sobre la jornada ampliada, es decir, una comparación acerca de la ampliación de la jornada escolar. Pero también y prioritariamente la investigación de Pascual (2012): ¿A les tres a casa? L’impacte social $i$ educatiu de la jornada escolar contínua, un estudio sobre la tendencia de organización del tiempo escolar, en el entorno europeo y en Cataluña. 


\section{pro.posıções}

\section{Marco teórico}

La dinámica de vida de las personas, en la actualidad, sugiere pensar con frecuencia en la necesidad de optimizar y ampliar el tiempo. En este caso, el tiempo se inserta como categoría relevante para la sociedad. Por eso es necesario mencionar que esta categoría es entendida como un constructo que se desarrolla bajo un determinado contexto social e histórico. Para Parente (2010, citado por Elias, 1998) el tiempo es un símbolo humano utilizado por un determinado grupo, a partir de instrumentos y procesos uniformizados para usar como cuadro de referencia y patrón de medida. De este modo es posible mencionar la existencia de varias tipologías de tiempo: tiempo laboral, tiempo escolar, tiempo de vacaciones, tiempo objetivo, tiempo subjetivo, etc.

Al tratarse del proceso educativo se advierte la existencia de varios aportes sobre la categoría tiempo escolar, que va desde el abordaje de conceptos (Leclerc \& Moll, 2012; Parente, 2010; Pascual, 2015;), pasando por la caracterización de estudios históricos, correlacionales, sociológicos, didácticos, comparados, etc. (Egido Galvez, 2011); y, por otros que vinculan el tema al desarrollo de diferentes acciones: planificación del aula, organización de la escuela para atender a las tareas y necesidades de estudiantes y profesorado, proposición de cambios e innovaciones, etc.

Respecto a la idea de innovación, por ejemplo, es posible insertar las categorías espacio y nuevas tecnologías de información y de comunicación en el análisis del tiempo, dada la configuración de nuevos sitios de aprendizaje, los ambientes virtuales. A pesar de considerar esa relevancia y el frecuente análisis de la categoría tiempo asociada a la categoría espacio, categorías interdependientes y complementarias, se privilegió, en este estudio, el tiempo como categoría suficiente y necesaria para situar el debate y proponer indagaciones para nuevos estudios.

El estudio en torno del tiempo escolar pasó a ganar mayor expresividad en las investigaciones en la década de 1990. Según Egido Galvez (2011, p.264) la publicación del estudio Prisioners of time, en 1994, en Estados Unidos, va a representar un hito para la organización del tiempo escolar, para ese país y toda Europa; posteriormente el tema también pasó a ser valorado en otros contextos. Más recientemente se advierte una producción frecuente de estudios, desde diferentes áreas y abordajes, debido a los intensos cambios en las sociedades. Por eso, el análisis de esta categoría sugiere pensar en la existencia de una diversidad de 


\section{pro.posıções}

\section{$e$-ISSN 1980-6248}

conceptos (distribución del tiempo de los estudiantes, trabajo de los docentes, tiempo que la escuela queda abierta, etc.), y en la comprensión sobre el contexto donde se desarrolla y el modo como se organiza.

Parente (2010, p. 144) menciona la existencia de diversos tiempos escolares. Y según ella estos tiempos pueden ser caracterizados en tiempo de escuela, tiempo de escolarización y tiempo en la escuela; el tiempo de escuela se refiere al inicio del proceso de escolarización, en que se presupone una edad inicial para el ingreso; el tiempo de escolarización, a su vez, se refiere al proceso de organización y sistematización de la enseñanza en grados y clases, con jerarquización de contenidos y etapas de enseñanza; diferente del tiempo en la escuela, puesto que se asocia al modo como cada sistema, cada escuela organiza el trabajo pedagógico. Y en este caso, hay que considerar la pluralidad de culturas, de valores y de concepciones que perfilan ese contexto (Parente, 2010). Por ello la autora afirma que es posible pensar en las múltiples posibilidades y alternativas a ser creadas en la escuela para atender a los colectivos de personas que allí interactúan (profesorado, alumnado, gestores, comunidad, etc.).

Respecto al tiempo diario de los estudiantes en la escuela, Pascual (2015, p.14) afirma que en los últimos treinta años se han producido reformas educativas en todo el mundo dirigidas a reorganizar y ampliar el tiempo de estada de los alumnos en los centros educativos; tiempo este que impacta sobre todo en la vida de ellos. Y como nos afirma el mismo autor, dicho tiempo se divide entre las horas de clase, el patio, las horas para comer, los deberes y actividades extraescolares (Pascual 2015, citado por Morán de Castro y Cruz López, 2011).

En base al concepto de tiempo escolar, considerando el tiempo diario de los estudiantes, este estudio privilegió la utilización del término jornada escolar, en la tentativa de garantizar una uniformidad en el mismo, dadas las diferentes terminologías utilizadas en los dos contextos. Y en el caso brasileño, es necesario mencionar que la doble jornada escolar puede ser de dos modos: en la escuela, con actividades en dos turnos o en otros espacios educativos, es decir, un turno de clases en la escuela y otro turno con actividades en espacios educativos fuera de la escuela, a diferencia de Cataluña que suele ocurrir, en general, en el mismo espacio educativo.

De un modo general, en la actualidad, el tema es objeto de debates y estudios, sobre todo por la promulgación de la Ley BR n. ${ }^{0}$ 13.415, de 16 de febrero de 2017, que reformuló la enseñanza secundaria, puesto que propone, entre otros aspectos, las clases en tiempo integral, es decir, la ampliación de la jornada diaria de los estudiantes de secundaria en los institutos. Y 


\section{pro.posıções}

de acuerdo con el informe de la Organización de las Naciones Unidas para la Educación, la Ciencia y la Cultura - UNESCO (2010), Brasil posee la menor jornada diaria de América Latina. En este sentido, el presente estudio se fundamenta en la idea de tiempo diario, de permanencia, de los estudiantes brasileños en los espacios educativos; tiempo este que presupone desafíos. Uno de ellos es el concepto, dado el panorama de terminologías y significados asociados al tema; educación integral o educación en tiempo integral; además de la compleja operacionalización de la propuesta de ampliación de la jornada regular.

El tiempo integral se refiere a la ampliación del tiempo regular de actividades educativas (que pueden ser intra escolares o extraescolares), es decir, contempla un tiempo mayor de permanencia del estudiante en la escuela/centro educativo. En cambio, educación integral sugiere la idea de educación para el desarrollo integral del estudiante, es decir, la educación debe abarcar todas las dimensiones de la vida (social, cultural, afectiva, etc.). Los embates sobre estas terminologías inducen a mencionar el Programa Mais Educação, creado por la Portaría Interministerial $\mathrm{n}^{\circ} 17 / 2007 \mathrm{y}$, posteriormente por el decreto $\mathrm{n}^{\circ} 7.083 / 2010$ del gobierno brasileño (Decreto BR, 2010). Este programa favorece la discusión e incentiva la propuesta de ampliación del tiempo escolar. Y corrobora la percepción de que el tiempo integral puede constituirse como un posible mecanismo para la educación integral.

Leclerc y Moll (2012, p.97) afirman que el Programa Mais Educação se constituye como una estrategia del gobierno central para inducir y fortalecer experiencias, así como ayudar, a la construcción de una agenda pública de educación integral a escala nacional.

Es evidente que la mera aprobación de una ley no garantiza que la misma se materialice como política educativa. Es necesario autonomía de la escuela para decidir sobre la ampliación de la jornada escolar. Sobre eso, es necesario considerar los distintos interlocutores/actores de esa acción, pues si por un lado su efectividad pasa por la idea de una política pensada y construida a partir de la voz de diferentes actores de la comunidad educativa (profesorado, gestores escolares, alumnado, etc.), por otro, hay que valorar la dinámica del federalismo brasileño, es decir, sobre las competencias y acciones de los entes federados (estado, municipios y gobierno central), lo que torna más difícil la implantación de esa propuesta.

Arretche y Schlegel (2014, p.4) afirman que una de las dificultades del federalismo brasileño reside en la fragilidad relativa de los estados, en cierta medida, explicada por la prevalencia de políticas homogéneas a ser adoptadas en todo el territorio nacional. Dicha 


\section{pro.posições}

estructura no favorece una gestión efectiva de la propuesta de ampliación de la jornada escolar en las diferentes instancias de gobierno, a pesar de los avances, en las dos últimas décadas, con la instauración del Programa Mais Educação. Por ello, dicha ampliación sugiere pensar inevitablemente que tiempo y espacio educativo son dos dimensiones relevantes en los estudios de currículo. El currículo, a su vez, no puede ser entendido al margen del contexto en el cual se configura ni tampoco independientemente de las condiciones en que se desarrolla (Sacristán, 2000). De esta manera, la reorganización de este tiempo presupone pensar en las estructuras de decisión de los centros educativos, es decir, reflexionar si esas estructuras son centralizadas o descentralizadas en la regulación y el control de las decisiones para atender a las necesidades de aprendizaje de los estudiantes, etc.

En el contexto español Herrero (2012, p.199) en su estudio pone énfasis en el concepto de jornada ampliada, categoría esta entendida a partir de otras dos categorías: jornada continua y jornada partida. Según el autor, la categoría jornada continua se refiere a las actividades desarrolladas en un único turno, mientras que jornada partida contempla los dos turnos, es decir, doble jornada (Herrero, 2012). En este sentido, el autor menciona que Bélgica, Bulgaria, Francia, Irlanda, Luxemburgo, Países Bajos y Reino Unido optaron por la jornada partida, es decir, un mismo curso tiene clases en dos turnos; mientras que España e Italia poseen los dos tipos de ofertas y permiten que los padres elijan el tipo de jornada de sus hijos (Herrero, 2012, p.199). Portugal, a su vez, presenta una tercera posibilidad, la jornada continua vespertina, es decir, la concentración de clases en el turno de la tarde; mientras que Grecia es el país que más se diferencia de los demás pues presenta dos posibilidades de oferta: jornada única por la mañana o jornada única por la tarde (p.199); esa es la oferta más frecuente en las escuelas públicas de Brasil.

La carga horaria semanal es concebida por Egido Galvez (2011, p.261) como el tiempo de actividades durante la semana; y en los países analizados, esta es más o menos homogénea en los años iniciales de la educación básica. En cambio, en España las clases finales presentan una carga horaria superior a los demás países (ver Tabla 1), además, es el quinto país europeo con mayor número de horas de enseñanza en la educación básica (Unesco, 2010, p.24).

Respecto al calendario de clases, es decir, la distribución de carga horaria semanal, en base al documento Organisation of school time in Europe (European Commission, 2016) España contempla un periodo de cinco días lectivos, en la semana, para la educación básica; y, con cerca 


\section{pro.posições}

de 175 días lectivos al año, siendo una carga horaria de 833 horas anuales en la educación primaria y 979 horas en la secundaria. Brasil, a su vez, el calendario oficial establece un periodo con cerca de 200 días lectivos al año, con un mínimo de 800 horas al año para la enseñanza primaria distribuidas en cinco días lectivos, en la semana.

Se constata que Francia, a diferencia de los demás países, ha reducido los días de clase, ahora los estudiantes frecuentan la escuela cuatro días por semana; los días miércoles quedan a los cuidados de familiares o de monitores en centros de ocio organizados por los ayuntamientos; servicio este pago por los padres, en proporción a sus sueldos (Egido Gálvez, 2011, p.15). A modo de síntesis, se presenta a seguir una tabla comparativa de la carga horaria semanal en diversos contextos.

Tabla 1: Carga horaria semanal de educación básica en países de la Unión Europea y Brasil

\begin{tabular}{|c|l|l|}
\hline \multicolumn{3}{|c|}{ Carga horaria semanal (total de horas) - Educación básica } \\
\hline Países & \multicolumn{1}{|c|}{ Clases iniciales } & \multicolumn{1}{c|}{ Clases finales } \\
\hline Brasil & $20 \mathrm{~h}$ & $24 \mathrm{~h} 50 \mathrm{~min}$ \\
\hline España & $25 \mathrm{~h}$ & $30 \mathrm{~h}$ \\
\hline Francia & $26 \mathrm{~h}$ & $23 \mathrm{~h}-24 \mathrm{~h}$ \\
\hline Italia & $27 \mathrm{~h}$ & $27 \mathrm{~h}$ \\
\hline Portugal & $25 \mathrm{~h}$ & $24 \mathrm{~h}-25 \mathrm{~h}$ \\
\hline
\end{tabular}

Fuente: Elaboración propia a partir de datos del estudio de Herrero (2012)

Los datos anteriores adquieren mayor representatividad al compararlos con datos de América Latina. De acuerdo con la Unesco (2010), los países de América del Norte y Europa Occidental presentan una media de horas de enseñanza oficial, acumulada durante los seis años de escolaridad, más alta que los países de América Latina; y en esta región los países que registran la mayor cantidad de horas, considerando los 9 años de escolaridad obligatoria, son, en este orden, México, Chile y Argentina. Brasil solo se sitúa por encima de la media, en América Latina, cuando se compara el número de días del calendario escolar: en Argentina y Uruguay el número de días lectivos es 180, en Chile es 190 y en Brasil 200 días (Unesco, 2010, p.17). Por ello, la reorganización de la jornada escolar, con vistas a la ampliación de actividades y presencia de los estudiantes, en las escuelas, sugiere pensar en el impacto de ese cambio para la vida social de los estudiantes y en los varios roles que esa institución viene incorporando, a lo largo de su historia, para proponer nuevas tareas a la rutina de estos estudiantes. 


\section{pro.posıções}

\section{La escuela pública brasileña y sus múltiples papeles}

El papel social de la escuela es frecuentemente analizado o cuestionado en estudios, debates, seminarios, conferencias, etc. Y a pesar del consenso de que la escuela es una institución que tiene como finalidad diseminar y producir saberes en la perspectiva de lanzar los fundamentos para el proceso de socialización, convivencia y supervivencia de la especie humana, parece que esta institución pasa a cumplir otras finalidades. Su papel viene cambiando a lo largo de los años. Al respecto, la historia de la educación evidencia que la escuela pública brasileña incorpora, cada vez más, actividades que buscan ocupar el tiempo del estudiante. Estas incorporaciones sugieren pensar, de inmediato, en una diversidad de aspectos: pedagógicos, culturales, sociales, etc.; y que a su vez se asocian a la puesta en marcha de la propuesta de ampliación de la jornada escolar.

Los cambios en el papel de la escuela, a lo largo de los años, están asociados al desarrollo de nuevas actividades, impulsados por la nueva configuración familiar y las desigualdades sociales, entre otros. En este sentido, es necesario mencionar que se observan en la Grecia Antigua, las primeras aproximaciones a conceptos de educación, e inicio de la relación educación y política. En dicha época se sitúa la perspectiva de educación como un enlace capaz de promocionar las condiciones necesarias para asegurar la continuidad del régimen del Estado, así como para formar al ciudadano en ciertas virtudes, es decir, propiciar el saber adecuado para la formación del carácter y del espíritu de los hombres.

En el período de la Edad Media, por su parte, los espacios educativos mantenían una estrecha relación con los principios y valores de la Iglesia y las clases ocurrían de modo individualizado; en el contexto brasileño, el proceso educativo de esa época fue marcado por la presencia masiva de los jesuitas (entre 1554 y 1759), los responsables de la organización de la primera escuela elemental del país. Sin embargo, en ocasión de la Constitución de 1824 se implantó la instrucción primaria gratuita y se organizaron los grados de enseñanza (escuelas de primaria, liceos, gimnasios y academias).

A fines del siglo XIX e inicios del siglo XX se percibe, en Brasil, en el contexto de la creciente expansión de la urbanización que la enseñanza básica pasa a "atender" al ideal modernizador de la sociedad. En ese ínterin, hay evidencias de avances significativos en la organización de la escuela: el paso de la enseñanza individualizada a la enseñanza colectiva, la 


\section{pro.posıções}

coeducación y la educación laica, entre otros aspectos. Sin embargo, es necesario evidenciar que tales logros fueron oriundos de un periodo marcado por discusiones efervescentes en torno a la necesidad de reforma de la escuela. Al respecto, se advierte la lucha entre dos movimientos: los defensores del movimiento renovador que propagaban una Pedagogía Nova; y el grupo de conservadores articulados a la Iglesia Católica. En ese contexto, el papel de la escuela fue cuestionado de modo incisivo, sobre todo con el Manifesto dos Pioneiros da Educação, en 1932, defendido por precursores como Anísio Teixeira, Fernando de Azevedo y Lourenço Filho, entre otros, intelectuales influenciados por las ideas de J. Dewey y H. Kilpatrick.

Ese manifiesto proponía principios como la educación pública, obligatoria, laica, gratuita y democrática. Y como resultado de ese movimiento, se constata posteriormente la puesta en marcha de una propuesta de ampliación de la jornada escolar desarrollada por Anísio Teixeira, que se basaba en la perspectiva de una formación lo más completa posible. Este concebía una educación que abarcase la ampliación de la cultura, la socialización y la formación para el trabajo; y por ello proponía un tiempo mayor en la escuela, la ampliación de sus funciones y su fortalecimiento como institución.

En la actualidad en Brasil, el Plano Nacional de Educação (2014-2024) (BRASIL, 2014) pone énfasis en la propuesta de ampliación de la jornada escolar como mecanismo para garantizar la igualdad de oportunidades educacionales a todos los estudiantes. En este sentido, ese plan parece sugerir nuevas actividades a ser incorporadas en la rutina de la escuela. Mas todavía, sugiere pensar que la escuela pasa a ejercer también el papel de protección social de niños y adolescentes en situación de mayor vulnerabilidad. De esta manera, el Programa Mais Educação presenta una propuesta que atiende en parte a esa realidad; puesto que propone el desarrollo de actividades para la jornada escolar ampliada. Según Cavaliere (2010), ese programa induce una reflexión acerca del papel actual de la escuela, en cuanto institución de enseñanza.

\section{Jornada escolar en el Distrito Federal (Brasil)}

El Distrito Federal es una de las veintisiete unidades federativas de Brasil, y posee cerca de tres millones de habitantes. Además de abrigar la capital del país, es una unidad federativa autónoma, que engloba características de municipio y estado, es decir, a ella son asignadas competencias de estos entes federados, pero con organización distinta de las demás unidades 


\section{pro.posıções}

del país, puesto que posee regiones administrativas denominadas de cidades-satélites gestionadas por representantes elegidos por el gobierno del Distrito Federal.

Ese distrito posee otra peculiaridad, recibe recursos del fondo constitucional que le asegura gastos con servicios públicos como educación, seguridad, salud, etc. (hecho que no ocurre con ningún otro estado). Tratándose de la educación, el gobierno de ese distrito está a cargo de la enseñanza básica de su territorio, es decir, controla, planifica, gestiona la educación (pública y privada), proclama leyes, etc.

Un aspecto interesante por mencionar es que el Distrito Federal promulgó una ley exclusiva: Ley BR n. ${ }^{\circ}$ 4.751, en 2012, que establece los fundamentos para la gestión democrática en los centros educativos. Otro aspecto diferencial es que ese distrito organiza las escuelas y colegios de un modo peculiar que permite la oferta de educación infantil, de primaria y de secundaria en espacios exclusivos, a diferencia de algunos estados del país, en los que es posible constatar la coexistencia de clases de primaria y secundaria en un mismo centro educativo. Además, también oferta clases de doble jornada (denominadas de tiempo integral) con cierta peculiaridad.

En relación a la oferta de clases con tiempo integral (doble jornada), en el Distrito Federal, esta se relaciona a la experiencia de Anísio Teixeira; e incluso, hay evidencias de que en esta región ocurrió una de las primeras iniciativas de ampliación de la jornada escolar. Sin embargo, la primera experiencia exitosa de ese tipo en el país, ocurrió a partir del trabajo de Anísio Teixeira, en 1950, con la creación de la primera Escola Parque, en Bahía. Dicha escuela concentraba actividades en un periodo de siete horas diarias, para los estudiantes.

En la actualidad, las experiencias de jornada escolar de tiempo integral, del Distrito Federal hacen referencia al trabajo de Anísio Teixeira, pero se organizan también a partir de otro marco legal y teórico.

La Constitución Brasileña, el Estatuto da Criança e do Adolescente (ECA), la Lei de Diretrizes e Bases da Educação Nacional (LDBEN), nº 9.394/96, constituyen un marco regulatorio elemental, actual, para reflexionar y consolidar una propuesta de ampliación del tiempo escolar de los estudiantes. Del mismo modo hay que se mencionar el Decreto Presidencial n ${ }^{\circ}$ 7.083/2010 en el que se establece que la educación en tiempo integral es la jornada escolar con duración igual o superior a siete horas diarias, durante todo el periodo lectivo, comprendiendo el tiempo total 


\section{pro.posıções}

\section{$e$-ISSN 1980-6248}

en que el estudiante permanece en la escuela o en actividades escolares en otros espacios educativos.

De ese marco se rescata que la organización de la jornada escolar, en la enseñanza primaria, tanto de centros públicos como privados de Brasil, obedece a lo previsto en la LDBEN, es decir, al cumplimiento de 800 horas anuales de actividades lectivas efectivas. De este modo, las escuelas suelen organizar las clases con una duración de 4 horas diarias y una media de 20 horas semanales. Sin embargo, en el Informe del Censo Escolar de 2010 del Instituto Nacional de Estudos e Pesquisas (2010) se constata que, en el caso de Brasilia, la media alcanza las 5 horas diarias, con 25 horas semanales.

Sostener una propuesta de ampliación del tiempo escolar presupone costes elevados y planeamiento en mediano y largo plazo. Por ello, la ampliación de esa política ocurrió de modo progresivo, a lo largo de los años, a partir de la creación del Fundo de Manutenção e Desenvolvimento da Educação Básica e Valorização dos Profissionais da Educação (FUNDEB), en 2007 (Brasil, 2007) que pasó a ofrecer una partida presupuestaria a las escuelas. En la actualidad, el FUNDEB subsidia el sistema público con recursos para que los estudiantes permanezcan más tiempo en los centros educativos. Pero es en el Plano Nacional de Educação (PNE), de 2014, donde se encuentra la delimitación de las acciones y las cifras a ser alcanzadas con esa propuesta.

En la Meta 6 del PNE, por ejemplo, está prevista la oferta de educación en tiempo integral en al menos el 50\% de las escuelas públicas, de forma que se atienda, por lo menos, al $25 \%$ de los estudiantes de educación básica. En conformidad con esa meta es necesario revisar algunos documentos para analizar la viabilidad y la complejidad de esa ampliación, y conocer como eso ocurrió en Brasilia.

Uno de los documentos relevantes para este estudio fue la investigación desarrollada por el Ministério de Educação do Brasil (MEC, 2010), iniciada en 2008; en la que consta la participación de 22 municipios, 18 estados de las cinco regiones geográficas del país. Por considerar la extensión de la investigación, y el objeto de análisis de este texto, se optó por centrar la atención en la experiencia de la región centro-oeste, específicamente el Distrito Federal.

El informe mencionado hace referencia a ideas preconizadas por Anísio Teixeira: educación pública, gratuita e integral, etc. Ideas estas que inspiraron la creación de la Secretaria de Estado Extraordinária para a Educaşão Integral del Distrito Federal (DF), en colaboración con la 


\section{pro.posıções}

\section{$e$-ISSN 1980-6248}

Secretaria de Estado de Educação; lo que permitió la implantación de la Educação Integral (EI) en el sistema de enseñanza pública de ese territorio. Esa secretaría movilizó el sistema de enseñanza local con el propósito de obtener el apoyo de los centros de educación primaria; y en 2009 fueron creadas las directrices conceptuales y de funcionamiento de la política de educación integral.

El Informe registra también, respecto al Distrito Federal, que la investigación se desarrolló en tres escuelas: la Escola Classe Itapeti, el Centro Educacional 01 Candangolândia y el Centro de Ensino Fundamental Arapoanga (CEF Arapoanga). Este último oferta enseñanza primaria (séries iniciais do ensino fundamental) y se ubica en Planaltina, región administrativa periférica de la capital, cuya mayoría de residentes posee bajo poder adquisitivo. Es necesario mencionar que, en el año 2008, este centro desarrolló actividades regulares y de tiempo integral, en el turno escolar opuesto, de modo que los estudiantes permanecían nueve horas en la escuela (entraban a las 9h, a las $12 \mathrm{~h}$ había un intervalo para la comida y salían a las $18 \mathrm{~h}$ ).

En la Escola Classe Itapeti, localizada en la zona rural de Brasilia, la propuesta de educación integral fue desarrollada entre 2008 y 2009, en base a la experiencia del municipio Pato Branco, Paraná. Las clases y las actividades se desarrollaban los días lunes y martes, de $6 \mathrm{~h} 30$ a 16h, y miércoles, jueves y viernes de $6 \mathrm{~h} 30$ a $17 \mathrm{~h} 30$. Dicha propuesta contemplaba la oferta de tres comidas diarias a los estudiantes que frecuentaban las actividades extraescolares (por ejemplo: informática, educación artística, ping-pong y clases de refuerzo).

El Centro Educacional 01 Candangolândia fue creado como centro de referencia en tiempo integral del Distrito Federal y poseía una organización diferenciada para atender al proyecto, es decir, no ofertaba enseñanza reglada sino que actuaba solamente como soporte pedagógico (en el turno diurno), con clases de refuerzo de portugués, matemáticas, ciencias naturales y ciencias humanas a alumnos de tres escuelas públicas. En el turno nocturno, desarrollaba actividades para estudiantes de secundaria, es decir, preparación para el Exame Nacional do Ensino Médio (ENEM).

La investigación del MEC apunta que en las escuelas donde fueron desarrollados los programas federales: Mais Educação y Escola Aberta había un acuerdo de colaboración entre el gobierno central, el gobierno del Distrito Federal y la Unesco; y que preveía la presencia de un equipo compuesto por un coordinador pedagógico y varios monitores para desarrollar el programa. Para Cavaliere (2010), la relación entre aquellas instituciones evidencia la 


\section{pro.posıções}

corresponsabilidad entre entes federados en la implantación del programa. Sin embargo, dicha relación también registra dificultades estructurales y de recursos humanos que impactaron en el desarrollo del programa (Brasil, 2010, p.148, Estudo Qualitativo).

Una de esas dificultades mencionadas fue la coexistencia de ofertas formativas diferenciadas, en un mismo espacio escolar, es decir, algunas actividades conflictuaban con las actividades regulares de la escuela.

En 2012 la Secretaría de Educación del Distrito Federal (SEDF) inició el proceso de implementación de la propuesta de tiempo integral en la totalidad de las escuelas que habían empezado el programa, es decir, se amplió la oferta de clases con jornada diaria de 7 horas. Las escuelas que habían empezado esta actividad pasaron a ofertar actividades en el turno escolar opuesto al 100\% de los matriculados en tres centros de educación infantil. Siendo que en 2013 y 2014 esa oferta fue implantada en otros espacios; y en 2014 fue implantada la primera etapa del Projeto Cidade Escola Candanga: educaşão integral, en veintiuna escuelas de educación básica, de la región administrativa de Brazilândia, es decir, en 17 escuelas del área urbana y 4 del área rural, en la perspectiva de atender a los estudiantes por cerca de 7, 8 o 10 horas diarias (SEDF, 2014).

Por medio de una observación realizada, en una escuela pública de Brasilia, se constató que la jornada escolar sigue la dinámica de clases de primaria con aulas durante cuatro días en la semana, en una misma escuela; y un día los estudiantes participan de actividades extras, en el turno escolar opuesto, en una de las Escuelas Parque de la ciudad. Paralelo a eso, se constató que la Secretaria de Educación del Distrito Federal está desarrollando una propuesta para implantar también la doble jornada en algunos colegios de secundaria.

A diferencia del caso del Distrito Federal, se identificó una experiencia de educación de tiempo integral en espacios fuera de la escuela. La investigación desarrollada por Rosa (2011) contempla una iniciativa de articulación entre escuela y comunidad, en el municipio de Nova Iguaçu, basada en el proyecto Ciudades Educadoras. Dicha experiencia se desarrolló a partir de acuerdos con instituciones asociadas para la concretización del programa de tiempo integral denominado de Bairro-Escola. Este programa fue desarrollado en escuelas públicas del sistema de enseñanza municipal, mediante una articulación con instituciones públicas y privadas, para realizar de actividades diversas en el turno escolar opuesto (Rosa, 2011, p.51).

A partir de las reflexiones y experiencias internacionales presentadas anteriormente, se indaga en la experiencia del Distrito Federal. Parece que el tiempo de estada del estudiante en el 


\section{pro.posıções}

turno escolar opuesto evidencia una paradoja: en el pasado hubo sobrecarga de tiempo diario del estudiante en la escuela y en la actualidad se evidencia un tiempo casi insuficiente para la realización de actividades que promueven el desarrollo global de los mismos, sobre todo porque en el turno escolar opuesto predominan actividades de recreación. Por otra parte, los profesionales que se encargan de esas tareas, en la actualidad, de un modo general, no son efectivos de los centros, lo que supone pensar en frecuentes cambios de personal; hecho que impacta en la calidad del proceso educativo.

Otro aspecto a considerar es que la práctica de tiempo integral, en espacios distintos de la escuela, no se vincula a las prácticas de las escuelas regulares; y tampoco evidencia la existencia de participación de la comunidad externa, como asociaciones de padres y madres. Por ello se entiende que es necesario valorar la opinión de los diferentes actores del proceso educativo en la construcción y ejecución de una propuesta como esta. En este sentido, los relatos sugieren pensar en la necesidad de ampliar la jornada escolar por medio de una articulación mayor entre actividades extraescolares y enseñanza regular. Al mismo tiempo, pensar en la necesidad de desarrollar nuevos estudios e investigaciones (de tipo transversal o longitudinal) que permitan conocer de hecho los resultados efectivos de esa propuesta, a partir de relatos de los estudiantes y de sus familias, y así indicar nuevas rutas de actuación.

\section{Jornada escolar en Cataluña (España)}

La Comunidad de Cataluña es una de las diecisiete comunidades del estado español que se dividen en comarcas y municipios; y posee cerca de 7,5 millones de habitantes. Es una de las comunidades más ricas e industrializadas del territorio español; e históricamente ha tenido una clara vocación de autogobierno que la caracteriza como comunidad-estado.

La responsabilidad de la educación, entre otras, es de la Administración de la Comunidad de Cataluña. Sin embargo, el gobierno central de España es quien establece la ley nacional para algunos aspectos. Por ejemplo, la creación de la asignatura Educación para la Ciudadanía y los Derechos Humanos, en 2006, asignatura está inserta en los cursos de secundaria, en que se preveía una atención especial a la igualdad entre hombres y mujeres. Sin embargo, con el cambio de gobierno dicha asignatura sufrió modificaciones y pasó a dar atención al aspecto cívico. 


\section{pro.posıções}

En lo referido al tema jornada escolar, se constata que las experiencias y los embates sobre el tema, en España (particularmente en Cataluña) contribuyen para ampliar el debate en el territorio brasileño. Desde la década de 1990 la ampliación de la jornada escolar en el territorio español viene implantándose de forma progresiva. Sin embargo, al revisar la historia de la educación en Cataluña, se percibe que, al final del siglo XX, surgió un movimiento denominado Ciudad Educadora que influyó en la implementación de la doble jornada escolar de esa comunidad. Pero se tiene registro de que el Ayuntamiento de Barcelona, en 1979, empezó a planear una propuesta integradora de educación formal, no formal e informal en la ciudad, para todos los que en ella habitaban. La consolidación de esa propuesta se materializó mediante un compromiso político que involucró a las familias, la escuela, el poder público, las empresas, etc.

Maragal (2008, p.15) afirma que el movimiento Ciudad Educadora, surge en Barcelona, cuando la ciudad se encontraba inmersa en un proceso de reconstrucción urbana - la ciudad salía de un largo periodo caracterizado por la ausencia de democracia; y en ese contexto, se proclamaba la necesidad de un urbanismo educado, respetuoso con el pasado histórico, más empeñado en proyectar la ciudad hacia el futuro. $\mathrm{Al}$ mismo tiempo, se observan otros aspectos que contribuyeron para la implementación de la política educativa de doble jornada escolar, en aquella época, como la intensificación de nuevas formas de organización familiar, el incremento de la inserción laboral de las mujeres, altas tasas de inmigración, las olimpíadas de 1982, etc.

Sin embargo, en 2013, la reducción significativa de los recursos destinados a la educación y la aprobación de una nueva normativa: Ley Orgánica para la Mejora de la Calidad Educativa (LOMCE), Ley no 08/2013 de ámbito nacional (España, 2013), conllevó cambios significativos en la organización de la educación en todo el país. Además de normativas de ámbito nacional, la Comunidad de Cataluña también se rige por leyes propias. Por ejemplo, la Ley de educación, $\mathrm{n}^{\mathrm{o}} 12$, de 6 de agosto de 2009, en la que se regula el sistema educativo de esa comunidad (Ley Cataluña, 2009).

Esa Ley contempla, entre otras competencias, facilitar pautas y referentes para la organización de la acción educativa y los contenidos de las enseñanzas y asegura que, en el marco de la autonomía de los centros, los proyectos educativos ordenen la gestión, la dirección, la organización pedagógica y los contenidos de las enseñanzas (Ley Cataluña, 2009).

El análisis de esa ley permite conjeturar que Cataluña posee cierta singularidad en la organización de la educación básica, es decir, comprende dos etapas: educación primaria (entre 


\section{pro.posıções}

los 6 y 12 años) y educación secundaria (entre los 12 años y 16 años), ambas de responsabilidad de la Administración de la Generalitat. Siendo que la educación infantil es de responsabilidad de la Administración Local (los ayuntamientos).

En lo referido a la enseñanza primaria es válido mencionar que la Administración Educativa de la Generalitat ejerce de titular de los centros públicos propios, y en tanto tal está a cargo de su gestión (determinación del calendario, de la jornada escolar, etc.). Asimismo, a la Administración Local (ayuntamiento) cabe la responsabilidad compartida en dichos centros educativos, es decir, participa en el gobierno de los centros educativos. Y entre otras tareas la Administración Local también es responsable de determinar la oferta educativa en su territorio, el proceso de admisión en los centros que prestan el servicio de educación, a través, si procede, de las oficinas municipales de escolarización. Además, se ocupa de las medidas que permiten a los centros llevar a cabo actividades extraescolares promovidas por la Administración de la Generalitat, así como la coordinación de dichas actividades.

En lo que respecta a la jornada escolar de educación primaria pública, es válido resaltar la particularidad de su organización, pues contempla dos tipos de jornada: continua y partida. En el caso de la jornada partida (doble horario), mayoritaria en las escuelas públicas de educación primaria de Cataluña, los padres tienen la posibilidad de elegir el número de días en que sus hijos van a participar en las actividades del turno escolar opuesto.

La organización de las clases suele ser de $9 \mathrm{~h}$ a 14h, por las mañanas, y de $15 \mathrm{~h} 30$ a 16h30, y las actividades extraescolares ocurren hasta las $17 \mathrm{~h}$ o $18 \mathrm{~h}$. Durante el tiempo libre, entre ambos turnos, los escolares tienen la posibilidad de almorzar en la escuela o ir a sus casas y regresar para las actividades extras (dos o tres veces a la semana). En lo atinente a la enseñanza secundaria obligatoria (última etapa de la educación básica), en los institutos públicos las clases se desarrollan en un único turno, es decir de $8 \mathrm{~h}$ a $14 \mathrm{~h}$, con intervalos de descanso entre las clases; esa práctica es denominada de jornada continua.

El estudio $i^{A}$ les tres a casa? L'impacte social $i$ educatiu de la jornada escolar contínua, de la Fundació Jaume Bofill (2012), presenta elementos contundentes sobre el tema. En base a la revisión de la literatura y de las experiencias y tendencias de países del entorno europeo, así como entrevistas con representantes del área de Educación de Cataluña, se constata que no hay evidencias, en investigaciones y experiencias de otros países y comunidades autónomas españolas, de que la jornada continua mejore el rendimiento académico de los estudiantes 


\section{pro.posições}

(Pascual, 2012, pp.14-15). Al contrario, se observa una acentuación de las desigualdades educativas (persistencia de resultados académicos bajos, dificultades de conciliación entre el horario escolar y el familiar; freno en el desarrollo profesional de las madres); sin mencionar el cierre de los centros educativos en el horario de la tarde (Pascual, 2012).

Por otra parte se registra que la jornada continua favorece a las familias en las que hay personas que no trabajan y por ello pueden recoger a los niños y llevarlos a casa para comer, lo que representa la supresión de los costes de comedor pues en las escuelas públicas catalanas los padres sufragan este coste y el de las actividades extracurriculares. Además, Pascual (2012, p.65) afirma que el $45,2 \%$ de las madres y el 72,1\% de los padres de Cataluña, con hijos adolescentes, regresan a casa después de las $18 \mathrm{~h}$ y que un $60 \%$ de los padres tiene dificultades financieras para pagar las actividades del turno escolar opuesto, de los hijos, en espacios fuera de la escuela.

Para Pascual (2012, p.40) el gobierno de Cataluña desarrolló un plan piloto de jornada continua, en clases de primaria, en el periodo 2012-2013, en respuesta a la demanda de centros educativos, impulsada por una parte de los docentes, es decir, ese plan atendió a la defensa de los intereses laborales de los profesores y con cierto nivel de acuerdo y oposición de padres y madres (en consonancia con las características propias de cada centro y de su entorno territorial, social y económico). Está en marcha la propuesta de ampliar ese programa piloto a otros centros públicos, para atender a la solicitud de centros que afirman que las clases en un único horario favorecen un mejor aprendizaje.

En base a datos internacionales, el estudio registra que la cantidad de horas lectivas no influye de modo sustancial en la obtención de resultados, pero sí en la calidad o el tipo de actividad realizada en ese horario (Pascual, 2012, p.15). Por otra parte, se expone que los estudiantes que se someten a una jornada continua están más fatigados al mediodía que los estudiantes de jornada partida (Pascual, 2012). El debate en torno a la ampliación de la jornada escolar, en Cataluña, está muy lejos del fin.

En la observación realizada en una escuela pública de primaria de Cataluña se constató que, a pesar de los aspectos positivos de la doble jornada, el desarrollo de actividades extraescolares está disociado de la enseñanza regular.

Por otra parte, es necesario mencionar que la mayoría de las comunidades autónomas sigue el camino al revés, es decir, una creciente ampliación en la oferta de la jornada continua. En este caso, la opinión del profesorado juega un papel preponderante, es decir, apoya la 


\section{pro.posıções}

concentración del aula en un solo turno - opción que le favorece; y más aún al gobierno local, dada la significativa reducción de los costes con la educación. Pero la ausencia de un fondo específico como subsidio (de almuerzo y pago de las tasas de actividades) para la permanencia del estudiante en el turno escolar opuesto quizás sea la cuestión más crucial para ese contexto.

\section{Jornada escolar en Cataluña y en el Distrito Federal: elementos de comparación}

La jornada escolar puede ser comprendida por medio de la distribución del tiempo a lo largo, del día y de la semana en la escuela. En el caso de Cataluña, la jornada escolar reglada de las escuelas públicas prevé el desarrollo de las clases en dos turnos, es decir, las actividades del turno escolar opuesto están previstas en el currículo escolar. En cambio, en el Distrito Federal predomina la oferta de clases en jornada única, es decir, un único turno.

En cuanto al total de horas semanales, la carga horaria es casi la misma en las clases iniciales (educación primaria) de los dos contextos, a diferencia de la educación secundaria: Cataluña contempla una carga horaria mayor en la secundaria y solo oferta en jornada única. En el Distrito Federal la secundaria también se oferta en jornada única, a pesar de la aprobación de una ley federal, reciente, que incentiva a los institutos a actuar en doble jornada (tiempo integral).

En cuanto a la organización de la jornada doble (partida), se observó que ambos contextos desarrollan actividades extracurriculares en el turno escolar opuesto. En el caso de Cataluña, esto ocurre todos los días de la semana, en la propia escuela; y las actividades frecuentes son: prácticas deportivas, danza, idiomas, refuerzo, etc. Hay un reclamo por parte de algunos padres para que se aumente ese tiempo para coincidir con su tiempo de estada en el trabajo. En el caso de las escuelas con oferta de tiempo integral (jornada doble), en el Distrito Federal, las actividades del turno escolar opuesto ocurren todos los días en otros centros, que se organizan para recibir estudiantes de una determinada escuela, cada día, es decir, estas actividades también están disociadas de la enseñanza reglada.

Por otra parte, el papel del gobierno, en los dos contextos, parece ser una cuestión clave para la participación de los estudiantes en esas actividades. Los estudiantes catalanes que desean permanecer en los centros todo el día, tienen que costear tanto el comedor como las actividades 


\section{pro-posıções}

extraescolares (excepto aquellos que poseen becas). En el Distrito Federal, costes son de responsabilidad del gobierno local. Sin embargo, los recursos financieros son provenientes del gobierno.

El análisis de la categoría elección de la ampliación permite afirmar que ese es el elemento más contundente del estudio. En Cataluña, el profesorado ha influido de modo significativo a la hora de decidir si se amplía o no la jornada escolar mientras que, en el Distrito Federal, la decisión se produce por determinación gubernamental. Además, en Cataluña, los padres y las madres se organizan mediante asociaciones y juegan un papel fundamental en esa dinámica, algo de lo que no hay constancia en el Distrito Federal. De este modo, se rescata una síntesis de las categorías analizadas, en los dos contextos, en la Tabla 2.

Tabla 2: Organización del tiempo escolar del estudiante de escuela pública, del Distrito Federal y de Cataluña

\begin{tabular}{|c|c|c|c|c|}
\hline Países & $\begin{array}{c}\text { Horas } \\
\text { diarias } \\
\text { Clases } \\
\text { regulares }\end{array}$ & $\begin{array}{c}\text { Jornada } \\
\text { escolar }\end{array}$ & $\begin{array}{c}\text { Calendario } \\
\text { escolar }\end{array}$ & $\begin{array}{c}\text { Calendario } \\
\text { lectivo } \\
\text { (año) }\end{array}$ \\
\hline $\begin{array}{l}\text { Distrito } \\
\text { Federal }\end{array}$ & 4,8 horas & $\begin{array}{c}\text { Continua/ } \\
\text { Partida }\end{array}$ & 5 días & 200 días \\
\hline Cataluña & 5 horas & $\begin{array}{c}\text { Continua/ } \\
\text { Partida }\end{array}$ & 5 días & 180 días \\
\hline
\end{tabular}

Fuente: elaboración propia a partir de Pascual (2015), Eurydice (2016) y Egido Gávez (2011).

A pesar de la aparente semejanza respecto a la organización del tiempo escolar, entre los dos contextos, se constata que las diferencias residen efectivamente en la opción por la ampliación de la jornada, así como la infraestructura para el desarrollo de esta. En los dos contextos, el Estado juega un papel distinto en esa dinámica social; mientras que uno establece un tiempo mayor de estada del estudiante en los centros (el caso de Cataluña, donde el coste recae cada vez más sobre la sociedad; el otro (el caso del Distrito Federal), que, a pesar de no atender a un número mayor de estudiantes con doble jornada, aun es responsable del bienestar social de los estudiantes.

Otro aspecto a registrar es que la propuesta de implantación de la doble jornada, en Cataluña, se desarrolló bajo un proceso colectivo, es decir, hubo un reclamo de padres y madres 


\section{pro.posıções}

y también por efecto del movimiento ciudad educadora, que se basaba en principios de que las escuelas debían ser gestionadas por la ciudad, por el municipio, por las familias y el profesorado. Se creía que las ciudades educan (Maragal, 2008).

Sin embargo, hay que valorar una diferencia muy clara entre los dos contextos: en el Distrito Federal es el gobierno central quien efectivamente propone la oferta de tiempo integral, es decir, garantiza a las escuelas la posibilidad de ampliar la jornada escolar por medio del Programa Mais Educação, mientras que, en Cataluña, el gobierno mantiene esta propuesta, a partir de la intervención y/o participación del profesorado, de padres y madres en defensa de los derechos de sus hijos, participación esta llevada a cabo regularmente por medio de una asociación. Sin embargo, Enguita (2000) afirma que el movimiento asociativo es, en el país, por esencia, un movimiento débil por cuestiones estructurales e históricas. Pero, es cierto, que ese movimiento aun juega un papel importante en torno al tema jornada escolar, en aquel contexto, hecho que no se constata en el Distrito Federal y quizás tampoco en otras regiones de Brasil.

\section{Consideraciones finales}

El análisis comparativo de los dos contextos apunta diferencias en la organización del tiempo escolar, pero también en la autonomía e infraestructura de los centros educativos evaluados, aunque no sea evidente una diferencia significativa en la carga horaria diaria, regular, de las clases. Al mismo tiempo el estudio induce a la necesidad de planear la ampliación de ese tiempo, en escuelas públicas del Distrito Federal, considerando el contexto donde se realizará dicha propuesta y las necesidades formativas del colectivo de estudiantes. Según Herrero (2012, p.199) el factor tiempo es un elemento clave en la construcción del currículo escolar para la mejoría de la calidad educativa. En este sentido, es posible deducir que el tiempo actual previsto para actividades extraescolares, en el Distrito Federal, no atiende a las necesidades educativas de los estudiantes.

Por otra parte, es posible predecir que, tratándose de una propuesta para la enseñanza secundaria, como prevé la nueva normativa, es razonable pensar que esa es una tarea hercúlea. Atender a las necesidades formativas de adolescentes y jóvenes exige debate, acuerdos y consensos con ese público y con el equipo de los centros educativos, es decir, es necesario involucrar diferentes instancias gubernamentales y diversos actores (gestores escolares, padres 


\section{pro.posições}

y docentes) en la discusión y en la decisión sobre la implementación de esa oferta. Hay que valorar la autonomía de las instituciones de enseñanza en la definición de las formas y los modos para poner en marcha un cambio como ese, así como identificar las características y la capacidad operativa de los mismos.

Otra cuestión relevante, respecto al Distrito Federal y a Cataluña es que no se puede olvidar que las escuelas se insertan en un proyecto mayor de sociedad; el que apunta al camino de varias formas de jornada escolar, es decir, hay que planear clases en jornada única y jornada doble para atender a un público diverso, en base al principio de integración de los saberes escolares con la vida en sociedad; la ausencia de esa integración en nada favorece la superación de desigualdades educativas y sociales de los estudiantes. Se entiende el tiempo como una categoría social que ocupa un papel fundamental para la ampliación del capital cultural del alumnado. Se trata, en suma, de intensificar el tiempo de los que pueden ir más deprisa y de prolongar el tiempo de los que necesitan ir más despacio (Enguita, 2000).

A modo de conclusión, las investigaciones internacionales respecto al tema ampliación del tiempo de permanencia del estudiante en la escuela apuntan, entre otros aspectos, a que la implantación siguió dos caminos: las demandas sociales por presión de algunos sectores o resultantes de investigaciones sobre el tema (Egido Galvez, 2011). Por eso es necesario insertar otro aspecto: la opinión de distintos actores del proceso educativo. Para ello hay que considerar que no haya sobrecarga del tiempo escolar sobre el tiempo social del estudiante (sus múltiples tiempos), su desarrollo integral presupone valorar también el tiempo social fuera de la escuela. Por ello se afirma la necesidad de profundizar ese tema por medio de otros estudios con énfasis en la idea de pensar otra forma de organizar el tiempo escolar, puesto que en los días actuales dicha organización sigue la referencia del siglo XIX, cuando las clases se organizaban en turnos de cuatro horas. 


\section{pro.posıções \\ $e$-ISSN 1980-6248}

\section{Referencias}

Arretche, M., \& Schlegel, R. (2014). Os estados nas federações: tendências gerais e o caso brasileiro. BID - Banco Interamericano de Desenvolvimento. Documento para Discussão IDB-DP334. Recuperado el 12.03.2018 de: https://goo.gl/u4YAs2

Brasil (2014). Plano Nacional de Educação (2014-2024). Brasília, Brasil: MEC. Recuperado el 12.03.2018, de: https://goo.gl/dwT8D6

Brasil (2010). Educação Integral/ Educação Integrada E(M) Tempo Integral: concepções epráticas na educação brasileira - mapeamento das experiências de jornada ampliada no Brasil. Brasilia: MEC/SECAD. Recuperado el 12.03.2018, de: http://educacaointegral.mec.gov.br/biblioteca.

Brasil (2010). Educação integral/ educação Integrada e(m) tempo integral: Concepções e práticas na Educação brasileira. Mapeamento das experiências de jornada escolar ampliada no Brasil: Estudo Qualitativo. Brasilia: MEC/SECAD. Recuperado el 12.03.2018, de: https://goo.gl/hFNfnR

Cavaliere, A.M. (2010). Questões sobre uma proposta nacional de gestão escolar local. In Anais IX Espaço Público da Educação: emergência de políticas e práticas de gestão local, regional e nacional. São Paulo, Brasil: ANPAE, 2010.Recuperado el 12.03.2018, de: https://goo.gl/KqiTo8 Egido Galvez, I. (2011). Los tiempos escolares en los sistemas educativos: análisis de algunas reformas recientes. Revista Española de Educación Comparada, 18, 255-278. ISSN: 11378654.

Elias, Nobert (1998). Sobre o tempo. Rio de Janeiro: Jorge Zahar Editor.

Enguita, M. F. (2000). La hora de la escuela: análisis y valoración de los procesos, los efectos y las opciones de la implantación de la jornada continua. Salamanca: Universidad de Salamanca.

European Commission/EACEA/Eurydice. (2016). The organisation of school time in Europe. Primary and secondary general education - 2016/17 school year. Eurydice Facts and Figures. Luxembourg: Publications Office of the European Union. Recuperado em 12.03.2018 de: https://goo.gl/WzB2oD 


\section{pro.posıções}

http://dx.doi.org/10.1590/1980-6248-2017-0095

$e$-ISSN 1980-6248

Fairbrother, G. (2015). Enfoques quantitatvos e qualitativos em educação. In M. Bray, B. Adamson, \& M. Mason (Orgs.), Pesquisa em educação comparada: abordagens e métodos (pp.101-126). Brasília: Liber Livro.

Franco, M.L. (2008). Análise de conteúdo. Brasília: Liber Livros.

Herrero, L.L. (2012). La perspectiva social de la jornada escolar en la unión europea: Estudio comparado en Italia, Francia, Portugal y España. Revista Española de Educación Comparada, 19, 193-218. DOI: http://dx.doi.org/10.5944/reec.19.2012.7583. Recuperado el 12.03.2018, de: http:// revistas.uned.es/index.php/REEC/article/viewFile/7583/7251

Leclerc, G. F. E., \& Moll, J. (2012). Educação integral em jornada diária ampliada: universalidade e obrigatoriedade? Em Aberto, 25, 17-49. DOI: 10.1590/S0104-40362016000100010. Recuperado el 12.03.2018, de: https://goo.gl/h6xXjI

Maragal, P. (2008). La ciudad de las personas. In Asociación Internacional de Ciudades Educadoras. Educación y vida urbana: 20 años de ciudades educadoras. In X Congreso de la Asociación Internacional de Ciudades Educadoras (pp.15-18). Barcelona: Santillana. Recuperado el: 12.03.2018 de: https://goo.gl/XJUkvb

Mason, M. (2015). Comparações entre lugares. In M. Bray, B. Adamson, \& M. Mason (Orgs.), Pesquisa em educação comparada: abordagens e métodos (pp. 127-168). Brasília: Liber Livros.

Morán, M.C. y Cruz, L. (2011). Vida cotidiana, tiempos escolares y derechos de la infancia. Educación Social. Revista de intervención socioeducativa, 47, 84-94.

Valentini, D. J.; Menegatti, Edna . As ações sociais na escola de tempo integral. In: Élsio José Corá; Adriana Salete Loss; Sérgio Begnini. (Org.). In Contribuições da UFFS: para educação integral em jornada ampliada. Chapecó/SC: Editora da UFFS, 2012, p. 391-406.

Parente, C. M. D. (2010). A construção dos tempos escolares. Educaşão em Revista, 26(2), 135156. https://dx.doi.org/10.1590/S0102-46982010000200007

Pascual, E.S. (2012). A les tres a casa? L'impacte social i educatiu de la jornada escolar contínua. Dossier de Imprenta. Barcelona, España: Fundació Jaume Bofill. Recuperado el 12.03.2018, de: https://www.fbofill.cat/sites/default/files/565-doc.pdf . 


\section{pro.posıções}

\section{$e$-ISSN 1980-6248}

Pascual, E. S. (2015). Escola a temps complet: cap a un model d'educació compartid. Informes Breus, 59. Barcelona, España: Fundació Jaume Bofill. Recuperado el 12.03.2018, de: https://goo.gl/fTkbPG

Rosa, A.V.N. (2011). Educação integral e(m) tempo integral: Espaços no Programa Bairro - Escola. Tesis de Maestría, Universidade Federal do Rio de Janeiro, Nova Iguaçu. Recuperado el 12.03.2018 de https://goo.gl/NM4TOs.

Sacristán, J.G. (2000). O currículo: uma reflexão sobre a prática (3a ed., E. Fonseca, Trad.). Porto Alegre: Artmed.

Schneider, S., \& Schimitt, C.J. 1998. O uso do método comparativo nas Ciências Sociais. Cadernos de Sociologia, 9, 49-87

Secretaria de Educação do Distrito Federal. (2014, novembro). Relatorio de gestão e políticas públicas 2011-2014. SEDF, Brasilia. Recuperado el 12.03.2018, de: goo.gl/0eKwfC

Unesco. (2010). Estado del arte: Escolaridad primaria y jornada escolar en el contexto internacional. Estudio de casos en Europa y América Latina. Buenos Aires, Argentina: Unesco. Recuperado el 12.03.2018, de: https://goo.gl/RC2ipc

Vega Gil, L. (2011). Educación comparada e internacional:proceso históricos y dinámicos globales (128pp.). Barcelona: Octaedro.

\section{Legislação}

Decreto BR n. ${ }^{\circ}$ 7.083, de 12 de janeiro de 2010. (2010). Dispõe sobre o Programa Mais Educação. Presidência da República. Casa Civil. Subchefia para Assuntos Jurídicos. Diário Oficial da União de 27.1.2010. Recuperado el 12.03.2018 de: https://goo.gl/uQ8kwa

Lei BR n. ${ }^{\circ}$ 9.394, de 20 de dezembro de 1996. (1996). Estabelece as Diretrizes e Bases da Educação Nacional. Diário Oficial da União, seção 1, ano CXXXIV, n. ${ }^{\circ} 248$. Recuperado el 12.03.2018 de: http://www.planalto.gov.br/ccivil_03/LEIS/L9394.htm Lei BR n. ${ }^{\circ} 11.494$, de 20 de junho de 2007. (2007). Regulamenta o Fundo de Manutenção e Desenvolvimento da Educação Básica e de Valorização dos Profissionais da Educação - FUNDEB. Diário Oficial da União de 21.6.2007 e retificado em 22.6.2007. Recuperado el 12.03.2018 de: https://goo.gl/4UKJwJ 


\section{pro.posıções}

Lei DF n. ${ }^{\circ}$ 4.751, de 7 de fevereiro de 2012. (2012). Dispõe sobre o Sistema de Ensino e a Gestão Democrática do Sistema de Ensino Público do Distrito Federal. Câmara Legislativa Do Distrito Federal. Diário Oficial do Distrito Federal n. 29 de 08/02/2012. Recuperado el 12.03.2018 de: https://goo.gl/z1tMx2

Lei BR n. ${ }^{\circ}$ 13.415, de 16 de fevereiro de 2017. (2017). Altera as Leis $n^{\circ}$ s 9.394, de 20 de dezembro de 1996, que estabelece as diretrizes e bases da educação nacional, e Lei 11.494, de 20 de junho 2007. Recuperado em 12.03.2018, de: http://www2.camara.leg.br/legin/fed/lei/2017/lei-13415-16-fevereiro-2017-784336norma-pl.html.

Ley Cataluña n. ${ }^{\circ}$ 12, de 10 de julio, de Educación. (2009). Recuperado el, 12.03 .2018 de: https://goo.gl/791uRy.

Ley Orgánica España 08/2013, de 9 de diciembre. (2013). Para la mejora de la calidad educativa. BOE, $n^{\circ} 295$, de 10 de diciembre de 2013, pp.97.858 - 97.921.

Submetido à avaliação em 20 de maio de 2017; revisado em 08 de março de 2018; aceito para publicação em 15 de abril de 2018. 